\title{
POLARIMETRIC COHERENCE OPTIMIZATION FOR INTERFEROMETRIC DIFFERENTIAL APPLICATIONS
}

\author{
Luca Pipia $^{1,2}$, Xavier Fabregas ${ }^{1,3}$, Albert Aguasca ${ }^{1}$, Carlos Lopez-Martinez ${ }^{1,3}$, Jordi J. Mallorqui ${ }^{1,3}$ \\ ${ }^{1}$ Remote Sensing Lab. (RSLab), Universitat Politècnica de Catalunya (UPC),Barcelona, Spain \\ ${ }^{2}$ Institut Cartogràfic de Catalunya, Parc de Montjuïc, Barcelona,Spain \\ ${ }^{3}$ Institut d’Estudis Espacials de Catalunya IEEC-CRAE/UPC \\ E-mail: luca.pipia@tsc.upc.edu/luca.pipia@icc.cat
}

\begin{abstract}
In this paper, the potentials of polarimetric coherenceoptimization techniques for differential interferometric SAR (DInSAR) applications are examined. For this purpose, the cutting-edge approaches available in the literature are considered. First, synthetic PolSAR data simulating homogeneous distributed scatterers are employed to demonstrate the convergence of the optimized differential phase to the deformation phase information. Then, real X-band ground-based PolSAR acquisitions concerning an urban environment are analyzed. The relation between optimum coherences and corresponding optimum phase in terms of deformation on retrieval is carefully analyzed using two zero-baseline fullypolarimetric data sets. In the end, general conclusions about the advantages and drawbacks of the alternative maximization approaches are drawn.
\end{abstract}

Index Terms - PolInSAR, ground-based SAR, DInSAR.

\section{INTRODUCTION}

The two main factors limiting the performance of any advanced differential interferometric SAR (DInSAR) technique are the number of trustful points within the monitored area and the quality of the corresponding phase information. Two main criteria are available in the literature for the estimation of the pixels' quality: the amplitude dispersion and the coherence stability. In the first case, the quality of the phase information along the whole interferograms stack is associated to the amplitudebased dispersion index $\mathrm{D}_{\mathrm{A}}$. The second approach invokes the ergodicity and the spatial homogeneity of the scattering process and estimates the accuracy of the interferometric phase information by spatial correlating each pair of stack data sets. Essentially, the higher the interferometric coherence, the more reliable the interpretation of the corresponding phase in terms of scatterers' position in time. Owing to the lack of longtime collections of polarimetric satellite-SAR (PolSAR) data, the mathematical formulation of the techniques implementing both criteria has been limited to the singlepolarization case. In this paper, a first attempt to fulfill this is carried out. To the end of investigating the potentials of coherence-optimization for enhancing the quality of deformation process retrieval, a comparison of the three cutting-edge methods [1][2][3] available in the literature when applied to zero-baseline PolSAR acquisitions is presented in this paper. A briefly description of the different approaches is first given. Special attention is paid to the statistical hypothesis each optimization method relies on. In order to demonstrate the meaningfulness of the proposed analysis, the convergence of optimized differential phases to the deformation phase component is then shown with simulated PolSAR data. Afterwards, the advantage and drawbacks of the different optimization techniques are studied in detail using a pair of real zero-baseline PolSAR data sets acquired at X-band using the UPC ground-based SAR sensor. In order to carry out a meaningful comparison of the benefits each approach is able to provide, the initial quality of each image pixel is labeled according to the selection criterion proposed in [4]. Since its rationale is the selection, pixel by pixel, of the channel of the scattering matrix [S] showing the highest coherence value, this method will be referred to as High technique. An analysis of the dispersion of the optimized differential phases with respect to the expected deformation phase component is then carried out and the obtained results are finally discussed.

\section{COHERENCE OPTIMIZATION}

Let $\left[S_{1}\right]$ and $\left[S_{2}\right]$ be the scattering matrices associated to each pixel of two PolSAR data sets acquired at the time instant $t_{1}$ and $t_{2}$, respectively. Being $\vec{k}_{P}^{1}$ and $\vec{k}_{P}^{2}$ the corresponding Pauli's scattering vectors, under ergodicity and spatial homogeneity hypotheses of the scattering process, it can be defined a $6 \times 6$ Coherency matrix $\left[T_{6}\right]$ as

$$
\left[T_{6}\right]=\left\langle\left[\begin{array}{l}
k_{P}^{1} \\
k_{P}^{2}
\end{array}\right]\left[\begin{array}{ll}
k_{P}^{1 \dagger} & k_{P}^{2 \dagger}
\end{array}\right]\right\rangle=\left[\begin{array}{cc}
{\left[T_{11}\right]} & {\left[\Omega_{12}\right]} \\
{\left[\Omega_{12}\right.}
\end{array}\right]
$$

where $\langle\cdot\rangle$ and $\dagger$ denote the spatial average operation, and the Hermitian transformation, respectively, $\left[T_{11}\right]$ and $\left[T_{22}\right]$ are the polarimetric Coherency matrices of the two data sets, and $\left[\Omega_{12}\right]$ is a $3 \times 3$ complex matrix containing the 


\begin{tabular}{|c|c|c|c|c|c|c|c|c|c|c|c|c|c|c|}
\hline \multicolumn{5}{|c|}{$\left[T_{11}\right]$} & \multicolumn{5}{|c|}{$\left[T_{22}\right]$} & \multicolumn{5}{|c|}{$\left[\Omega_{12}\right]$} \\
\hline \multirow{3}{*}[D]{} & 0.92 & 0 & $0.2 \mathrm{e}^{\mathrm{j} \pi / 4}$ & & & {$[0.9$} & $0.05 \mathrm{e}^{\mathrm{j} \pi / 4}$ & $0.2 \mathrm{e}^{\mathrm{j} \pi / 4}$ & & & 0.88 & $0.1 \mathrm{e}^{\mathrm{j} \pi / 5}$ & 0 & \\
\hline & 0 & 0.7 & 0 & {$[D]^{\dagger}$} & {$[D]$} & $0.05 \mathrm{e}^{-\mathrm{j} \pi / 4}$ & 0.6 & 0 & {$[D]^{\dagger}$} & {$[D]$} & $0.1 \mathrm{e}^{-\mathrm{j} \pi / 5}$ & 0.4 & 0 & {$[D]^{\dagger}$} \\
\hline & $0.2 \mathrm{e}^{-\mathrm{j} \pi / 4}$ & 0 & 0.85 & & & $0.2 \mathrm{e}^{-\mathrm{j} \pi / 4}$ & 0 & 0.75 & & & 0 & 0 & 0.67 & \\
\hline
\end{tabular}

Table 1: Theoretical $\left[T_{11}\right],\left[T_{22}\right]$ and $\left[\Omega_{12}\right]$; the matrix $[\mathrm{D}]$ relates the Covariance $[\mathrm{C}]$ and the Coherency $[\mathrm{T}]$ matrices.

differential interferometric information relating the different polarization channels [4]. The estimate of the complex differential coherence, considering two generic scattering mechanisms (SMs) $\vec{u}_{i}$ and $\vec{u}_{j}$ is directly given by

$$
\gamma_{i j}\left(\vec{u}_{i}, \vec{u}_{j}\right)=\vec{u}_{i}^{\dagger}\left[\Omega_{12}\right] \vec{u}_{j} / \sqrt{\vec{u}_{i}^{\dagger}\left[T_{11}\right] \vec{u}_{i} \vec{u}_{j}^{\dagger}\left[T_{22}\right] \vec{u}_{j}} .
$$

Two main polarimetric coherence-optimization techniques based on $\left[T_{6}\right]$ analysis are available in the literature. The first one was proposed in [1] and solves the optimization problem by Lagrangian multiplier technique. The solution is represented by three pairs of scattering vectors providing three complex coherence values ordered as so that $\left|\gamma_{\text {opt } 1}\right| \geq\left|\gamma_{\text {opt } 2}\right| \geq\left|\gamma_{\text {opt } 3}\right|$. Since this method allows the selection of two different mechanisms at the end of the spatial or temporal baseline, i.e., it may be $\vec{u}_{\text {opt } 1} \neq \vec{u}_{\text {opt } 1}^{2}$, in the following this method is referred to as Different Scattering Mechanisms (DSM). Contrarily to $D S M$, the approach proposed in [2] constraints the two scattering mechanism to be identical. For this reason, in the following it will be referred to as Equal Scattering Mechanisms (ESM) method. In order to achieve a mathematical solution, ESM assumes that the two Coherency matrices $\left[T_{11}\right]$ and $\left[T_{22}\right]$ are very similar and reformulates the denominator of (1) as follows

$$
\widetilde{\gamma}_{i}\left(\vec{u}_{i}\right)=2 \vec{u}_{i}^{\dagger}\left[\Omega_{12}\right] \vec{u}_{i} / \vec{u}_{i}^{\dagger}\left(\left[T_{11}\right]+\left[T_{22}\right]\right) \vec{u}_{i} .
$$

It is worth noticing that, even if it is always $\left|\widetilde{\gamma}_{i}\right| \leq\left|\gamma_{i i}\right|$, the two coherence carry the same phase information. To note that optimization of $\widetilde{\gamma}_{i}$ is not analytically solvable and the solution is obtained using an iterative algorithm [2].

An alternative strategy for polarimetrically maximizing the differential coherence optimization was proposed in [3]. Despite it entails the condition $\vec{u}_{i}=\vec{u}_{j}$ as ESM, it solves the optimization problem at [S] level by sweeping all the possible combination of ellipticity/orientation angles $(\psi, \chi)$ defining the polarization state of the propagating wave. Its rationale is to look for the polarization basis transformation providing the highest among all the co-polar and cross-polar coherence values:

$$
\gamma_{o p t}=\max _{(\psi, \chi)}\left[\gamma_{x x}(\psi, \chi), \gamma_{x y}(\psi, \chi)\right] .
$$

The subscripts $x x$ and $x y$ denote the copolar and crosspolar channels of the generic scattering matrix achieved by the $(\psi, \chi)$-based polarization reference transformation, respectively. Since no statistical hypothesis is assumed about $\left[\mathrm{S}_{1}\right]$ and $\left[\mathrm{S}_{2}\right]$, the solution indeed corresponds to the highest coherence achievable with the constraint $\vec{u}_{i}=\vec{u}_{j}$. In general, multiple mechanisms are identified by different local coherence maxima [3]. The main drawback of the technique proposed in [3] is its computational inefficiency. In the remaining, this last method is referred to as SOM, standing for Subspace Optimum Method.

In the case of zero-baseline PolSAR acquisitions focused on differential applications, the main concern becomes the retrieval of the optimum phase that better describes the possible deformation process characterizing the monitored area. According to the model described in [4], the higher the coherence, the lower the effect of the scattering process' temporal decorrelation, and, consequently, the more correct the interpretation of the differential phase in terms of radial displacement. In the light of this reasoning, it becomes meaningful to look into the potentials of the abovementioned techniques to improve the quality of the retrieved deformation information.

\section{SIMULATED POLDINSAR DATA}

In order to assess the capability of the optimization methods in Section II, simulations are first employed. The algorithm proposed in [5] for the simulation of homogenous distributed targets has been used to generate two PolSAR data sets in the horizontal-vertical polarization basis. The theoretical polarimetric matrices characterizing the simulated distributed targets are reported in Table 1. Then, a deformation-phase component has been introduced by adding a common term $\phi_{\text {def }}$ to one PolSAR data set, namely the slave one. As the diagonal elements of $\left[\Omega_{12}\right]$ are real, the interferometric phase from $h h, h v$, and $v v$ channels converges to $\phi_{\text {def }}$, as it is shown in Fig. 1. Likewise, the optimized phase is expected to follow the same behavior as long as the corresponding coherence value increases. The plots in the sequence of images displayed in Fig. 2 confirm that the simulated distributions are generated correctly.The first image group (a) shows the mean value and the standard deviation of the coherence 

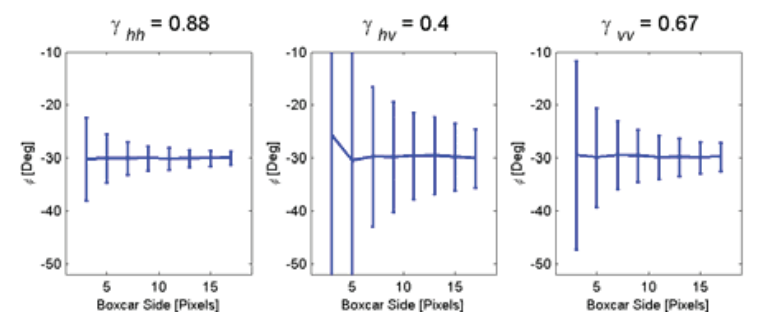

Fig. 1: Mean value and standard deviation of differential phase given by the simulated $h h, h v$ and $v v$ polarization channels as a function of boxcar size $\left(\phi_{\text {def }}=-30^{\circ}\right)$.
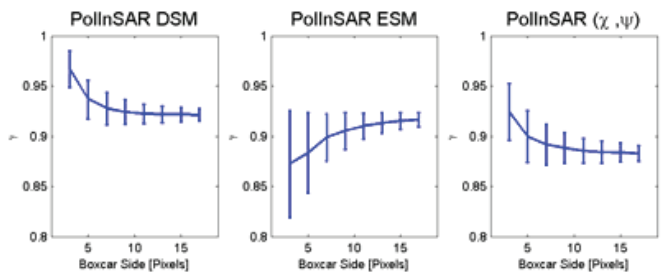

(a)
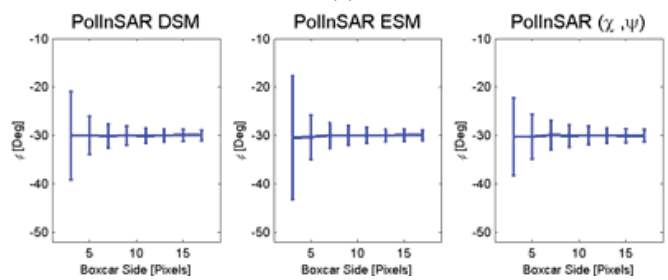

(b)

Fig. 2: Mean value and standard deviation of optimized coherences $\gamma$ (a) and phases $\phi$ (b) given by DSM, SOM, ESM as a function of boxcar size using simulated PolSAR data $\left(\phi_{\text {def }}=-30^{\circ}\right)$.

provided by DSM, ESM and SOM techniques using simulated data, as a function of the averaging boxcar size. It can be noted that DSM and ESM converge to the same optimum coherence. This should be expected, being the two Coherency matrices almost identical. On the contrary, the lower coherence value provided by $S O M$ is due to the $1^{\circ}$ angular step employed for $(\psi, \chi)$ angles in the optimization process.

The second image row shows the corresponding differential phases, which converges to a non-biased estimation of $\phi_{\text {def }}$ for the three approaches. Nonetheless, it is worth pointing out that DSM provides the best performance in terms of coherence optimization. Contrarily, stating if $S O M$ or ESM must be preferred is not straightforward. On the one hand, SOM is limited by the sampling step used for $(\psi, \chi)$-based optimization and the convergence of its phase estimation to the true phase information is slower. On the other hand, ESM better performance might be due to the fact that $\left[T_{11}\right]$ and $\left[T_{22}\right]$ are almost identical. When the hypothesis of stationary polarimetric behavior of the scattering process within the averaged area is not completely fulfilled, its convergence to the optimal solution might not be assured. This problem will be addressed in Section IV. Yet, the simulations clearly show the capability of the three different optimization techniques to reduce the uncertainty of the phase information extracted from homogeneous areas.

\section{REAL GBSAR POLDINSAR DATA}

In this section, two real PolSAR data sets acquired using an X-band gbSAR system, with a temporal delay between data of a month, concerning an urban environment are analyzed. The zero-baseline data sets were gathered in the framework of the Sallent measurement campaign driven by the Remote Sensing Laboratory of UPC from June 2006 to July 2007 [4]. According to the time scale of the subsidence phenomenon of a few centimeters per year in the radial direction [4], the one-month time span makes it possible to state that the deformation occurred in between the two acquisitions is negligible. Likewise, it is long enough to allow one to observe significant timedecorrelation effects within the urban environment.

The mosaic of differential coherence and phase in Fig. 3 shows the results provided by the polarimetric approaches described in Section 2 for a $7 \times 7$ averaging boxcar. For completeness, also the coherences given by the two copolar channels ( $h h$ and $v v$ ) have been displayed. A clear improvement of $|\gamma|$ is detectable for the three optimization techniques: DSM provides the highest coherence values, as expected, whereas $S O M$ seems to work better than $E S M$. The reason is likely to lie in the time different between $\left[T_{11}\right]$ and $\left[T_{22}\right]$, which makes ESM fundament hypothesis fail. Nonetheless, in terms of differential phase improvement, a brief inspection of the phase image collection reveals that no outstanding enhancement is obtained though differential coherence optimization.

In order to carry out a quantitative analysis scene's pixels have been grouped into three subsets depending on their High coherence value [4]: subset 1 contains pixels showing a value within ]0.8:1] range, subset 2 within ]0.6:0.8] range, and subset 3 within ]0.4:0.6] range. Then, the optimization methods have been applied to each subset using different boxcar sizes, namely $5 \times 5,7 \times 7$, and $9 \times 9$. A zero-mean differential phase distribution has been obtained in all the cases, confirming that any deformation process can be neglected between the two acquisitions. As a consequence, the standard deviation of the optimized phases constitutes a useful statistical descriptor of the real benefits provided by each optimization approach for differential application purposes. In fact, the higher the benefits, the lower the dispersion of pixel phase information around zero.

The results obtained in the different cases are summoned in the plots of Fig. 4. It can be observed that SOM always to improve the performance of the High selection criterion. Despite the higher enhancement of the absolute 


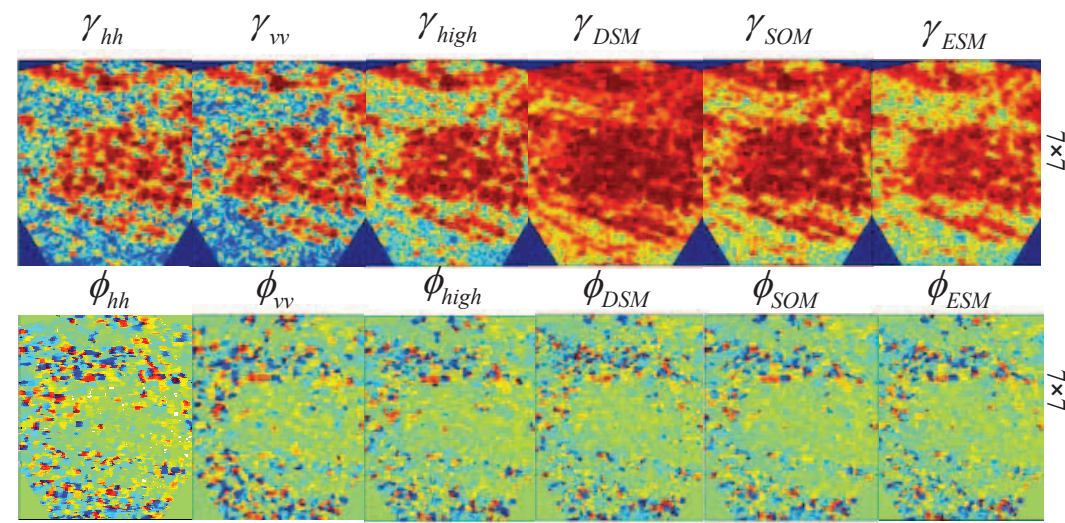

Fig. 3: Collection of differential coherence $\left(1^{\text {st }}\right.$ row $)$ and differential phase $\left(2^{\text {nd }}\right.$ row) images provided by the gbSAR zero-baseline PolSAR data sets.

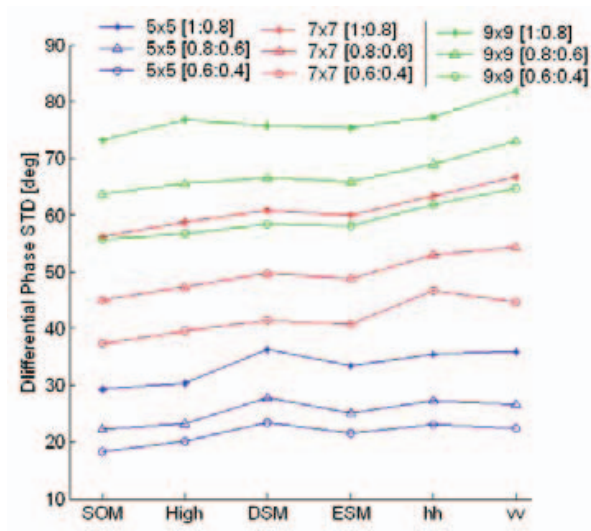

Fig. 4: STD of the differential phase STD provided by $h h, v v, H i g h, D S M, E S M$ and $S O M$ coherences. value coherence, DSM phase standard deviation is always higher than any the other two techniques.

The reason seems to lie in the selection of two SMs, which essentially tunes the way each averaged area is described so that the scattering processes become more resembling. Owing to the deterministic nature of the complex urban environment, the spatial homogeneity hypothesis is probably unfulfilled. As a consequence, the deformation information within the differential phase is likely to be corrupted by the range-shift of the equivalent phase centers that DSM carries out to maximize $|\gamma|$. On the contrary, the selection of a unique SM seems to be more suitable for this type of scenario, avoiding any phase center displacement due to the optimization process itself. Concerning ESM, it is supposed to work improperly due to time non-stationary of the Coherency matrices within the urban area, corroborating the conclusion drawn from the comparison of DSM and ESM coherence images in Fig. 3. Nonetheless, it is worth stressing both DSM and ESM provides a much larger differential phase dispersion than the High approach, meaning that the optimization process they perform worsens the estimation of the deformation phase component. Contrarily, SOM is able to improve a slight improvement of this estimation, corroborating the idea that the employment of the same SM seems to be more suitable to differential applications when spatial homogeneity hypothesis is not guaranteed within the observed area, as in urban area.

\section{CONCLUSIONS}

Simulations of homogeneous distributed areas have shown the convergence of the polarimetric optimization methods DSM, ESM and SOM to common deformation phase term. Their application to real gbsar data has stressed that the direct optimization coherence parameter does not necessarily lead to an improvement of the deformation estimation. Despite the noteworthy increase of the differential coherence obtained with the ESM and
DSM techniques, unsatisfactory estimations of the subsidence have been obtained. The unexpected results achieved using the ESM are related to the time nonstationary of the Coherence matrix describing the urban behavior. In any case, the mismatch between spatial homogeneity hypothesis and the deterministic heterogeneity characterizing the urban environment represents the most likely reason of the unsatisfactory deformation-rate estimation provided by DSM. Finally, the best results have been obtained using the SOM method. The employment of the same scattering mechanism for the description of the scatterers' behavior seems to constitute a compulsory condition for retrieving a reliable description of the deformation phenomenon.

\section{AKNOWLEDGMENT}

This work has been funded by the I+D Spanish project TEC2008-06764-C02-01 and the Ramon-y-Cajal program.

\section{REFERENCES}

[1] S.R. Cloude and K.P. Papathanassiou, "Polarimetric SAR Interferometry," IEEE Transactions on Geoscience and Remote Sensing, Vol. 36, No. 5, September 1998.

[2] E. Colin, C. Titin-Schnaider, and W. Tabbara, "An Interferometric Coherence Optimization Method in Radar Polarimetry for High-Resolution Imagery," IEEE Transactions on Geoscience and Remote Sensing, Vol. 44, No. 1, January 2006.

[3] L.Sagués, J.M. Lopez-Sanchez,J.Fortuny, X. Fabregas,A. Broquetas and A.J. Sieber, "Indoor Experiment on Polarimetric SAR Interferometry," IEEE Transactions on Geoscience and Remote Sensing,, Vol. 38, No. 2, March 2000.

[4] L. Pipia, X. Fabregas, A.Aguasca, C. López-Martínez, S. Duque, J.J Mallorqui, and J. Marturià, "Polarimetric Differential SAR Interferometry: First Results With Ground-Based Measurements," Geoscience and Remote Sensing Letters, Vol. 6, No. 1, January 2009.

[5] L. Pipia and X.Fabregas, "Generation of Pol-SAR and Pol-InSAR Data for Homogeneous Distributed Targets Simulation," POLINSAR 2005 Workshop, Frascati, January 2005, Italy 